\title{
Adaptive Deadline-aware Scheme (ADAS) for Data Migration between Cloud and Fog Layers
}

\author{
Adnan Khalid ${ }^{1}$ and Muhammad Shahbaz ${ }^{2}$ \\ ${ }^{1}$ Government College University, Lahore, Pakistan \\ [e-mail: adnan.khalid@gcu.edu.pk] \\ ${ }^{2}$ University of Engineering and Technology, Lahore, Pakistan \\ [e-mail:m.shahbaz@uet.edu.pk] \\ *Corresponding author: Adnan Khalid
}

Received October 16, 2017; accepted October 21, 2017; published March 31, 2018

\begin{abstract}
The advent of Internet of Things (IoT) and the evident inadequacy of Cloud networks concerning management of numerous end nodes have brought about a shift of paradigm giving birth to Fog computing. Fog computing is an extension of Cloud computing that extends Cloud resources at the edge of the network, closer to the user. Cloud computing has become one of the essential needs of people over the Internet but with the emerging concept of IoT, traditional Clouds seem inadequate. IoT entails extremely low latency and for that, the Cloud servers that are distant and unknown to the user appear to be unsuitable. With the help of Fog computing, the Fog devices installed would be closer to the user that will provide an immediate storage for the frequently needed data. This paper discusses data migration between different storage types especially between Cloud devices and then presents a mechanism to migrate data between Cloud and Fog Layer. We call this mechanism Adaptive Deadline-Aware Scheme (ADAS) for Data migration between Cloud and Fog. We will demonstrate that we can access and process latency sensitive "hot" data through the proposed ADAS more efficiently than with a traditional Cloud setup.
\end{abstract}

Keywords: computer networks, grid computing, Internet of Things, distributed computing, cache storage 


\section{Introduction}

When Cloud computing came forward as the next big thing the main attraction was the replacement of a fixed storage and compute system with a virtual environment that provided a pay-as-you-go service architecture. This meant that an organization could do away with the fixed cost of storing data on physical storage devices as well as save on the expenses incurred on in-house compute resources. This opportunity was unprecedented and hence a large amount of organizations, small, medium and large, around the world shifted from enterprise systems to Cloud based solutions for their storage and compute needs. As the number of users increased the Cloud started experiencing issues related to latency and security. With the emergence of Internet of Things (IoT) the world started to realize that the elastic storage capabilities of the Cloud computing paradigm had their limitations and that the dynamic resource allocation that gave the illusion that Clouds have unlimited storage was after-all dependent on the available bandwidth. IoT entails that everything in the world can eventually act as an end node and can transmit and receive data and information. This concept encourages an unprecedented growth in the data access points that interact with the Cloud networks and hence the issues of latency (especially when dealing with critical real-time data) become more evident. Cisco's Flavio Bonomi came up with the idea of an extension to Cloud computing to cope up with the challenges presented by latency sensitive data [1]. He named his proposed paradigm "Fog computing”.

\subsection{What is Cloud Computing?}

Cloud computing is a computing technology whereby both hardware and software resources are distributed over a network ensuring that the end user receives a highly scalable infrastructure on a pay as you go basis. The receiver of the Cloud could either be a software-as-a-service (SaaS) or platform-as-a-service (PaaS) provider or an immediate user of infrastructure. The essense of Cloud computing, however, lies in the fact the hardware and/or the platform is leased, scalable and flexible and is paid on a per usage basis. [2]

\subsection{What is Fog Computing?}

Fog computing is an extension of a technology called Cloud computing. It streches the resources provided by Clouds to the edge of the network, nearer and closer to the user. The purpose of adding Fog extension to Clouds is to reduce latency issues faced by real time application users. Cloud data centers store and process data at a centralized location far away from the user [3]. Due to many network-hops between the end user and the centralized location delays are caused, these delays cannot be overlooked for time-sensitive applications. This latency issue can be resolved by incorporating a Fog layer that constitues of highly virtual machines with ephemeral storage capabilities and is situated in close proximity to the end users. Thanks to its broad environmental sharing the Fog is well situated for real time computation [4]. Fog provides closer geographical proximity, fast storage and a highly distributed environment. Fog and Cloud mutually provide an environment that fulfills the requirements of time-sensitive and bulk data processing applications.[5] Fog model provides benefits in marketing, computing, entertainment and other applications[6]. It improves Quality of Service and reduces latency. 


\subsection{Why Moving from Cloud to Fog?}

As a matter of fact with every technology or every luxury that man enjoys for that matter, there is an opportunity cost associated with the flexibibility and scalablility offered by cloud networks [7]. Since the inherent nature of the cloud is geographically segregated and far away for the receiver and connectivity is achieved via an IP based network, accessing and processing data experiences a natural lag. Although this lag is not significant for many applications but could prove lifethreatening for time-critical applications where a delay of millisecond can mean a risk of life. Also with the future evidently heading towards Internet of Things it is safe to predict a huge rise in the number of cloud users which in turn would cause problems with bandwidth availability and subsequently increase the lag that is experienced by the real-time application receiver. The only ideal way forward seems in the direction of dispersing the storage and processing of clouds into a structure that allows storage and processing of real-time data separately while also sccomodating for bulk storage needs and analytics. This structure has been coined as the Fog by Flavio Bonomi and has been endorsed by CISCO [8].

Cloud systems are situated within the Internet, which is a huge diverse network with a mesh of different technologies, topologies with no inner control. Latency affects the value of services and Cloud cannot guarantee that how much time it will take to process the information and give the response. Time-critical applications are poorly affected by wait and jitter caused by latency in the network [9]. Therefore, a new platform is needed to address latency related issues and to allow real-time processing of data. The latest platform that gives a fresh set of web applications and facilities to the users, by enhancing the capabilities of the Cloud platform is known as Fog Computing.

\subsection{Our contribution}

We have over the course of this paper given a mechanism to migrate data from traditional clouds to Fog networks. Our work identifies the data that needs to be migrated based on its hotness and profiles data so that the migration is performed alongwith reasonable load balancing. We have evaluated the utility costs of both the data on clouds and Fog and have shown that the migration is truly beneficial.

Table 1. COMPARISON OF CLOUD COMPUTING AND FOG COMPUTING

\begin{tabular}{|l|l|l|}
\hline & \multicolumn{1}{|c|}{ Cloud Computing } & Fog Computing \\
\hline Target User: & $\begin{array}{l}\text { Common Internet } \\
\text { users }\end{array}$ & Mobile users \\
\hline Service Type: & $\begin{array}{l}\text { Global information is } \\
\text { collected world-wide }\end{array}$ & $\begin{array}{l}\text { Partial localized information } \\
\text { services linked to exact deployment } \\
\text { locations }\end{array}$ \\
\hline Hardware: & $\begin{array}{l}\text { Abundant and scalable } \\
\text { storage space \& compute } \\
\text { power }\end{array}$ & $\begin{array}{c}\text { Restricted storage, compute power } \\
\text { \& wireless interface }\end{array}$ \\
\hline
\end{tabular}




\begin{tabular}{|l|l|l|}
\hline $\begin{array}{l}\text { Distance from } \\
\text { Users: }\end{array}$ & $\begin{array}{l}\text { Far-away from } \\
\text { consumers } \\
\text { interconnect through IP } \\
\text { Network. }\end{array}$ & $\begin{array}{l}\text { In the physical contiguity and } \\
\text { interconnect through single hop } \\
\text { wireless connection }\end{array}$ \\
\hline $\begin{array}{l}\text { Working } \\
\text { Environment: }\end{array}$ & $\begin{array}{l}\text { Ware-house-size } \\
\text { building with central air } \\
\text { conditioning systems }\end{array}$ & $\begin{array}{l}\text { Outdoor (parklands, streets etc.) or } \\
\text { indoor (shopping malls, restaurants, } \\
\text { etc.) }\end{array}$ \\
\hline Deployment: & $\begin{array}{l}\text { Maintained and } \\
\text { Integrated by Google, } \\
\text { Amazon, etc. }\end{array}$ & $\begin{array}{l}\text { Distributed or Integrated in } \\
\text { regional areas by native business } \\
\text { (shopping Mall retailer, local } \\
\text { telecommunication vendor etc.) }\end{array}$ \\
\hline
\end{tabular}

\section{Migrating From A Traditional Cloud To Fog}

In order to benefit fully from the concept of Fog computing we have to devise a framework that would ensure a systematic extension of a Cloud environment to an environment that showcases a Cloud and a Fog layer. We have identified four major parts of the migration process namely Data shift, Application shift, Service shift and Security measures. A detail of the proposed steps for each shift is presented as follows.

\subsection{Data Shift}

This step of the migration process begins with the identification of different end-node devices as Fog storage devices. Routers, sensors, terminals and other devices that interact with the cloud can be used in a localized network to form a layer between the end user and the cloud network. This layer is called the fog layer.

The process of shifting data from these fog devices to the cloud network and vice versa requires differentiation of data on the basis of its "hotness". Data is considered hot when it is required for real time processing and requires a substantial number of Inputs and Outputs per second (IOPS).

\subsection{Application Shift}

Application shift will take care of deploying distributed applications at the client and server ends with one part of the application handling the real-time processing on the Fog device and the other part handling bulk processing and storage on the cloud device. Both these parts would have to be integrated and synchronized to insure both accuracy of real-time results and maintenance of integrity of bulk data stored at the cloud end. It is imperative that some sort of mechanism is devised that would determine the workload sharing of the real-time and the bulk module of the application.

\subsection{Service Shift}

This part of the migration framework would provide service architecture models for IaaS, PaaS and SaaS. A separate paper proposing these models for Fog computing is in the 
publishing process. One of these models is used in this paper later on.

\subsection{Security Meausures}

Cloud security has been the most talked about area of research in cloud computing. The public cloud has faced severe criticism because of its fragile security. Fog computing provides a solution by providing some sort of control over the distribution and proximity of critical data. It is however necessary to employ techniques like PKI and CA to actually make the Fog networks a much more secure alternative to cloud computing.

This paper discusses the first part of the migration framework which addresses data migration between Clouds and Fog. This data migration would include a determination of the kind of storage devices that would be available at the Fog layer and their comparison with the Cloud storage devices. It will then be concerned with some sort of a mechanism that can differentiate data on the basis of latency sensitivity and data temperature. Finally an Infrastructure-as-a-service model for Fog computing would be used to help demonstrate how the to and fro movement of data would take place between Clouds and Fog.

\section{Data Migration between Cloud and Fog}

\subsection{Internet of Things (IOT) and the Cloud}

The rise of the Internet of Things concept has rendered the Cloud storage mechanism as inefficient because the number of nodes interacting with the Cloud has increased exponentially [10]. This increases amount of load on the Cloud network and exposes the limitations of bandwidth available to Cloud networks.[21] This calls for data profiling through which data can be identified as critical, latency sensitive and "hot".

\subsection{Data Profiling}

In order to migrate the correct amount and type of data from Cloud to Fog and vice versa we need to ditinguish data according to some parameters. With Fog computing the main concern regarding to data access and processing is latency. This means that one parameter that must be taken into account when determine what data to move to the Fog layer should be latency sensitivity. In this regard another aspect of data that needs attention is its temperature.

\subsubsection{Data Temperature}

Data can be deemed "hot" or "cold" depending on its usage. A "hot" data would typically be data that has to be processed within a certain time frame. As the time passes the temprature of "hotness" cools down and the data becomes less critical, resulting in the term "cold" data. At a given instance determining "hot-spots" in data that is stored can be aided by IO profiling whereby IOPS-intensive data is moved to the faster Fog layer. A key challenge in this regard is faced from the fact that hot-spots in data keep changing over time i.e. cold data that is scarcley accessed suddenly or gradually becomes hot due to it being frequently accessed or becoming performance critical as a result of a certain event. These fluctuations in the nature of data necessasitates an adaptive mechanism that can evaluate the future needs of the system and migrate appropriate block of data from Cloud to Fog and vice versa. 


\subsubsection{Deadline}

Data selection for migration requires preemptive determination of hot or latency-sensitive data. Such determination requires screening data for certain indications such as criticality and IOPS-sensitivity.[12] One important aspect of this screening is determining a reasonable deadline within which the migration ould complete in order to meet the deadline of workload to which the "hot" data is associated.

\subsection{Adaptive deadline aware system for Data Migration (ADAS)}

Migration from Clouds to Fog and vice versa is constrainted by deadlines. Data that is "hot"at a particular time might get cold over time and hence it is important to perform migration within the deadline that is associated with the workload that includes hot data. To migrate data under such a constraint some assumptions are necessary.

\subsubsection{Assumptions for Adaptive-deadline-aware migration}

- $\quad$ The I/O workloads are cyclical in nature and sets of different workload types alternate between each other within a given time period, say every $24 \mathrm{hrs}$.

- $\quad$ Data heat has to be constantly monitored to determine the hot data extents.

- $\quad$ Fog storage devices exhibit scarcity in terms of per unit cost.

- Data migration for each workload has a certain deadline.

\subsubsection{Utility cost for storage}

Since the main concern for a shift to a distributed Fog-Cloud environment is a reduction in latency [11], the main performance measure that needs to be monitored and optimized has to be the response time.

Let $\mathrm{t}$ be the response time during time period $[\mathrm{t} 1, \mathrm{t} 2]$ then the response time function for a multi-tiered Fog environment can be denoted as,

$$
f(t), t \in[t 1, t 2]
$$

The utility cost of the whole Fog environment it terms of the response time needed for communication between the Fog devices and the Cloud network can be represented as the sum of all response times over a time period of $[\mathrm{t} 1, \mathrm{t} 2]$ such as,

$$
U_{t 1, t 2}(t)=\int_{t 1}^{t 2} f(t) d t
$$

Let $\mathrm{w}$ denote workload cycle and $\mathrm{p}$ denote the average peak response time and $\mathrm{U}_{0}$ denote the utility cost when no migration takes place then,

$$
U_{0}=p \times \mathrm{w}
$$


Let $U_{r}$ be the utility rise as a result of data migration. It can be represented in the following way,

$$
U_{r}=U_{0}-U
$$

Our goal is to keep the minimum utility cost while migrating within a guaranteed deadline. In case the migration does not happen, the average peak response time is maintained.

\subsubsection{Basic Migration model}

The basic migration model that we propose contains three phases as shown in Fig. 1.

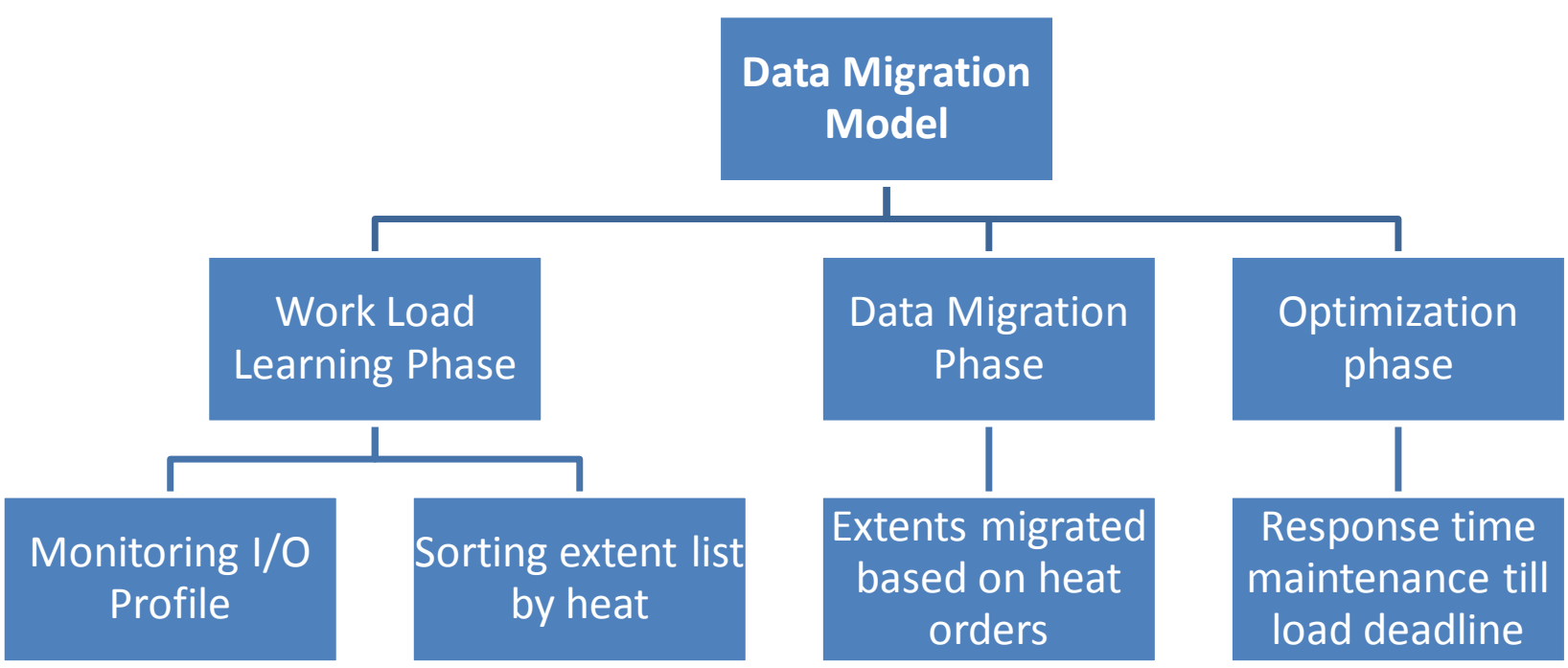

Fig. 1. Basic model for migration of data from cloud to Fog

As shown in Fig. 1, the work load learning phase continuously monitors the I/O profile and sorts the data according to its heat. The extent lists are created such that the hotter data is accessed first. If the learning phase takes $\mathrm{X}$ time then for this phase the utility would be,

$$
U=\int_{0}^{x} f(t) d t
$$

The second phase is the data migration phase. Here the hot data extents are migrated in order of their heat. Since the number of hot extents is limited in any given workload, each new hot extent yields a smaller reduction in response time. A time comes eventually when the impact of migration is saturated and the response time can no futher be reduced. This point can be called the point of convergence and this leads eventually to the average response time. 
If the data migration phase takes y time then utility cost of this phase is,

$$
U=\int_{x}^{x+y} f(t) d t
$$

After the convergence point arrives, the optimization phase would start and here the response time is maintained at the stable minimum until the deadline is achieved.

If the optimization takes $\mathrm{z}$ time then the utility cost of this phase is,

$$
U=\int_{x+y}^{x+y+z} f(t) d t
$$

The workload cycle time for an individual workload would equal the sum of time lengths of all the three phases and can be represented as,

$$
\begin{aligned}
& w=x+y+z \\
& U=\int_{0}^{w} f(t) d t
\end{aligned}
$$

The utility cost of an individual workload for a single migration between the Fog and the Cloud layer can be represented as,

$$
U=\int_{0}^{x} f(t) d t+\int_{x}^{x+y} f(t) d t+\int_{x+y}^{x+y+z} f(t) d t
$$

The peak response time achieved $\mathrm{p}$ and the saturation response time $\mu$ give us the data migration response time function,

$$
f(t)=\frac{\mu-p}{x} \mathrm{x} t+p, t \in[x, x+y]
$$

The response time function for one cycle of migration between Fog and Cloud would then be,

$$
f(t)=\left\{\begin{array}{l}
p, t \in[0, x] \\
\frac{\mu-p}{x} \mathrm{x} t+p, t \in[x, x+y] \\
\mu, t \in[x+y, w]
\end{array}\right\}
$$


Hence in light of the above representations we can finally calculate the utility cost of a single migration between Cloud and Fog layers to be,

$$
U=\int_{0}^{w} f(t) d t=(p-\mu)+\left(\frac{1}{2} \mathrm{x} \mathrm{y}^{+} x\right)+\mu \mathrm{xw}
$$

To actually see the benefits of this data migration in terms of reduction in utility cost and hence a faster response time we need to calculate the utility rise i.e.

$$
\begin{aligned}
& U_{r}=p \times \mathrm{Xw}-\left((p-\mu)+\left(\frac{1}{2} \times \mathrm{y}+x\right)+\mu \times \mathrm{xw}\right) \\
& =(p-\mu) \times\left(w-x-\frac{1}{2} \times y\right)
\end{aligned}
$$

It is evident from discussion above that utility cost differential would definitely establish the efficacy of migrating data from Cloud networks to Fog environments. All we need now is an architecture that would employ the above theorems.

\section{Data Migration between Cloud and Fog}

Now with the emergence of Fog computing the IaaS for Fog would have a distributed structure that would bifurcate real-time data processing and analytical processing also insuring separate bulk storage and ephemeral storage of data. Our proposed model is as follows and it is the first of its kind.

Fig. 2 distinctly shows that with the eminent real time nature of use of the IoT applications the Fog services would need insuring the availability of two kinds of networks for storage. The Fog layer would be providing ephemeral storage meaning that the real time latency sensitive data would be stored temporarily, processed quickly with a response time ensure that the access is truly real time. 


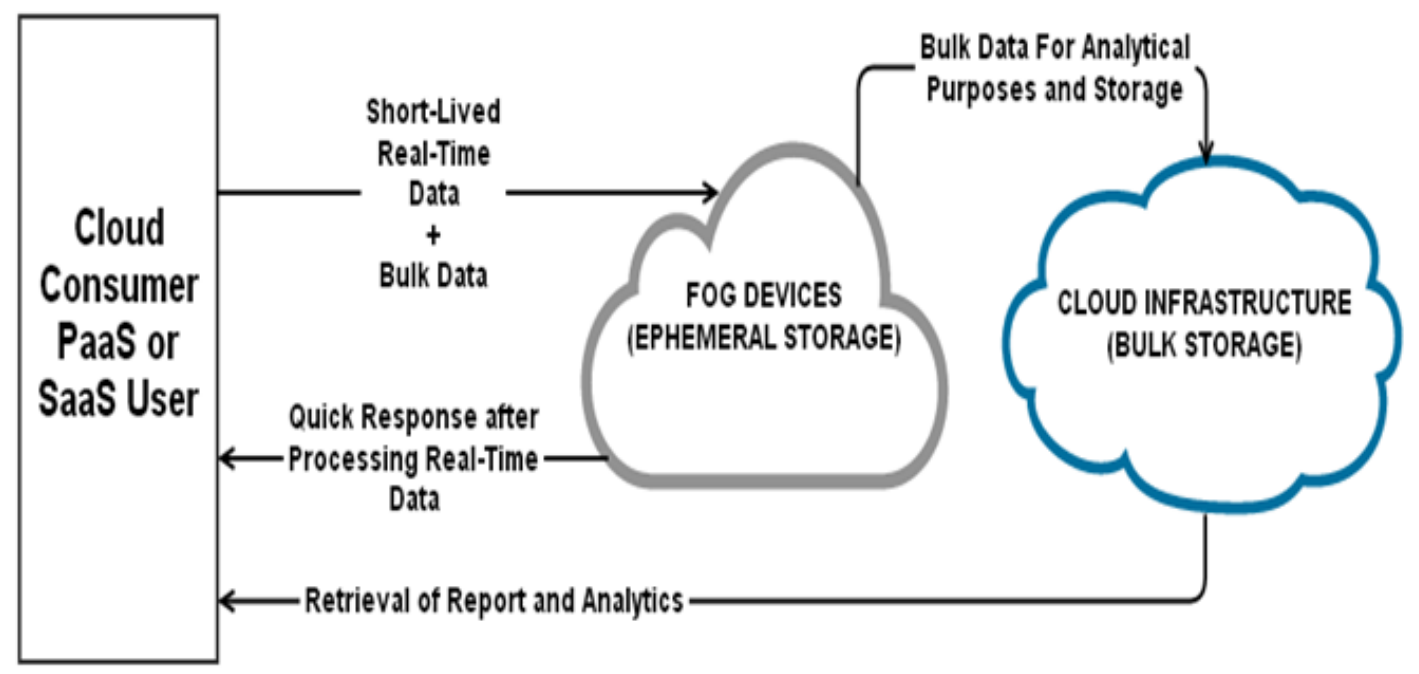

Fig. 2. Infrastructure as a Service (IaaS) for Fog

A cloud layer would provide bulk storage for data and would support heavy storage devices capable of storing big amounts of data. This data can also help generate reports for analytical purposes.

Once our ADAS and this brand new architecture for Fog implementation are combined we can easily calculate the reduction in utility cost and the resultant reduction in response time and hence substantiate that data migration between Fog and Cloud networks would lead to lesser lags and faster access of data.

\section{Discussion and Conclusion}

This paper serves two vital purposes as far as research into migrating systems from Cloud networks to the more novel Fog environments is concerned. Firstly, following the discussion about how Fog networks are able extensions of Cloud networks we put forward a mechanism ADAS that can be employed to determine the utility cost of storage and the reduction in it due to migration. This we believe is the best measure of the response time reduction that can help determine that migrating data to Fog devices would reduce latency. Secondly, we provide an architecture that would effectively represent the distributed environment that the Fog proposes. This service architecture is the first of its kind and combined with the ADAS gives a complete structure of how to migrate data between Clouds and Fog. We believe that the future of Fog computing would lie in this adaptive migration of hot data from Fog to Cloud and vice versa.

Currently, appropriate pricing for cloud services is an issue that the data center owners are constantly trying to answer. With the advent of the IoT and the increase in the usage of cloud services, it is crucial to strike a balance between the profits made by the data center owners and the utility provided to their tenants. The tenants who are the main end users of the cloud services increasingly require fast paced, real-time processing and compute resources leading 
to more cost for the cloud service providers. The cloud providers are pushed to use storage mediums that are faster and more expensive than the traditional HDD. To shift entirely to mediums such as SDD would both be expensive and risky as the SDD is not only 4 times the cost of HDD but also presents the problem of data decay.

ADAS, the scheme that we propose solves this issue for the cloud provider beautifully and hence would be a corner stone in the efforts to provide affordable, fast paced, real time Fog services to the end user.

Some of the services that can heavily benefit from ADAS in terms of efficient processing of real-time and critical data are as follows.

\subsection{Smart traffic lights and connected vehicles}

Smart traffic lights (STL) and connected vehicles have the prospective to renovate the way traffic is controlled on the roads.[16] Interoperable wireless network comprising vehicles, traffic signals, cell phones, and various smart devices have the ability to avoid collisions, maintain traffic flow and issue warning in case of any risk approaching [13] [19].

The fog attributes are ideal for this scenario as fog devices (nodes) can sense environmental data through sensors locally detecting any pedestrian/biker approaching and speed of vehicle [14]. It can further interact with adjacent lights to maintain traffic flow [15]. Based on this information, fog devices can locally issue warnings to vehicles and alter its cycle of green lights to avoid collisions. Fog Architecture provides all the essential features such as low latency, geo distribution, mobility, safety and analytics and location-awareness. Real-time analytics are achieved on the basis of data gathered by STL nodes and long-term analytics is processed by data sent to cloud by fog devices. [17][18]

\subsection{Decentralized smart building}

The provision of control and decisions practice through sensors is a promising way of enhancing energy efficiency in smart building. In these buildings wireless sensors are used to measure environmental entities such as humidity, air, temperature, levels of various gases. The reading form all sensors on a specific floor are accumulated and then decision is made accordingly. Fog devices are employed to respond to the data collected, actions are then taken such as adjusting temperatures, reduce moisture and injecting fresh air, turning lights on/off(sensing movement)thus improving overall environment and conserving energy.

\subsection{Health monitoring}

As the society becomes increasingly mobile, deployment of wireless network has also progressed significantly. With this enhancement emergent healthcare applications can also prove to be beneficial making smart and optimized health decision. Pervasive Healthcare Applications is one such vision which claims healthcare to everyone, every time and everyplace irrespective of time, geographical location and other constraints. [20].

\subsection{Electoral Polling}

Conducting polls is a huge task in context to the data flow that takes place. Millions of people 
cast vote in the span of only a few hours and the integrity of their vote is of great value as it determines the fate of a nation. Manual voting can be easily tampered with or rigged and hence the need of automated polling is always there. With the concept of electronic voting come the issues of data integrity, data storage, data processing, result tabulation etc. A traditional computing environment would require fixed infrastructure and substantial investment to carry out the task and would still be less flexible than a cloud based solution. The cloud network however can face issues of data security and data loss. In this scenario an ADAS based Fog network would help immensely in the smooth conductance of E-polling.

Fog computing or fogging is by no means an alternative to cloud computing. In fact, fogging combined with the traditional cloud platform will provide an optimal computing platform for fast emerging IoT environment.

\section{Future Work}

The ADAS can be extended to have a look-ahead mechanism that would determine the hotness and coldness of data over a certain time. We can also see the emergence of intelligent Fog devices that would have the ability to identify critical data and route it to Fog devices or Cloud storage respectively. The use of agents to decide whether at a particular time a chunk of data needs to be processed at the fog layer would reduce the latency associated with cloud networks considerably as the process of data transfer will be dynamic. Moreover, if instead of fixed tagging of data as hold or cold a mechanism was to be introduced in the future to determine the hotness or coldness of data intelligently, and then the wastage of time that it takes to make the switch between cold and hot data would be reduced. As far as future is concerned, it lies in the developments in the IOT and the Fog [22]. It is therefore safe to say that there can and will be numerous opportunities for future researchers to use this research and develop on it.

\section{References}

[1] Bonomi, F., Milito, R., Natarajan, P., \& Zhu, J., "Fog computing: A platform for internet of things and analytics," Big Data and Internet of Things: A Roadmap for Smart Environments Springer, International Publishing, pp. 169-186, 2014. Article (CrossRef Link)

[2] Khalid, A., \& Shahbaz, M., "Cloud computing technology: services and opportunities," Pakistan Journal of Science, vol. 65, no. 3, pp. 348-351, 2013.

[3] Madsen, H., Burtschy, B., Albeanu, G., Popentiu-Vladicescu, F. L., "Reliability in the utility computing era: Towards reliable fog computing," in Proc. of 2013 20th International Conference on Systems, Signals and Image Processing (IWSSIP), IEEE, pp. 43-46, 2013. Article (CrossRef Link)

[4] Hong, K., Lillethun, D., Ramachandran, U., Ottenwälder, B., Koldehofe, B., "Mobile fog: A programming model for large-scale applications on the internet of things," in Proc. of Proceedings of the second ACM SIGCOMM workshop on Mobile cloud computing ACM, pp. 15-20, 2013. Article (CrossRef Link)

[5] Naranjo, Paola G. Vinueza, Mohammad Shojafar, Leticia Vaca-Cardenas, Claudia Canali, Riccardo Lancellotti, Enzo Baccarelli, "Big Data Over SmartGrid-A Fog Computing Perspective," in Proc. of SOFTCOM Workshop, pp. 1-6. 2016

[6] Weiss, A., "Computing in the clouds,” Computing, 2007. Article (CrossRef Link) 
[7] Dillon, T., Wu, C., Chang, E., “Cloud computing: issues and challenges,” in Proc. of 2010 24th IEEE international conference on advanced information networking and applications, pp. 27-33, 2010. Article (CrossRef Link)

[8] Bonomi, F., Milito, R., Zhu, J., Addepalli, S., "Fog computing and its role in the internet of things," in Proc. of Proceedings of the first edition of the MCC workshop on Mobile cloud computing, ACM, pp. 13-16, 2012. Article (CrossRef Link)

[9] Siemens, G., Long, P., "Penetrating the Fog: Analytics in Learning and Education," EDUCAUSE review, vol. 46, no. 5, 2011.

[10] Nemirovsky, M., Milito, R., \& Yanuzzi, M., "Fog computing," in Proc. of Class Conference ICREA/BSC, vol. 24, 2012.

[11] Zhang, G., Chiu, L., Liu, L., "Adaptive data migration in multi-tiered storage based cloud environment," in Proc. of 2010 IEEE 3rd International Conference on Cloud Computing, IEEE, pp. 148-155, 2010. Article (CrossRef Link)

[12] Naranjo, P. G. V., Shojafar, M., Abraham, A., Baccarelli, E., “A new Stable Election-based routing algorithm to preserve aliveness and energy in fog-supported wireless sensor networks," in Proc. of Systems, Man, and Cybernetics (SMC), 2016 IEEE International Conference on, IEEE, pp. 002413-002418, 2016. Article (CrossRef Link)

[13] Firdhous, M., Ghazali, O., Hassan, S., "Fog computing: Will it be the future of cloud computing?" in Proc. of The Third International Conference on Informatics \& Applications (ICIA2014), 2014.

[14] Peter, N., "FOG Computing and Its Real Time Applications."

[15] Stojmenovic, I., Wen, S., "The fog computing paradigm: Scenarios and security issues," in Proc. of Computer Science and Information Systems (FedCSIS), 2014 Federated Conference on, IEEE., pp. 1-8, 2014. Article (CrossRef Link)

[16] Fröhlich, B., Plate, J., "The cubic mouse: a new device for three-dimensional input," in Proc. of Proceedings of the SIGCHI conference on Human Factors in Computing Systems, ACM, pp. 526-531, 2000. Article (CrossRef Link)

[17] Baccarelli, E., Naranjo, P. G. V., Shojafar, M., Scarpiniti, M., "Q*: Energy and delay-efficient dynamic queue management in TCP/IP virtualized data centers," Computer Communications, vol. 102, pp. 89-106, 2017. Article (CrossRef Link)

[18] Varshney, U., "Pervasive healthcare and wireless health monitoring," Mobile Networks and Applications, vol. 12, no. 2-3, pp. 113-127, 2007. Article (CrossRef Link)

[19] Naranjo, Paola G. Vinueza, et al, "P-SEP: a prolong stable election routing algorithm for energy-limited heterogeneous fog-supported wireless sensor networks," The Journal of Supercomputing, vol. 73, no. 2, pp. 733-755, 2017. Article (CrossRef Link)

[20] Baccarelli, Enzo, et al, "Fog of Everything: Energy-efficient Networked Computing Architectures, Research Challenges, and a Case Study," IEEE Access, 2017. Article (CrossRef Link)

[21] Shojafar, Mohammad, et al, "FLAPS: bandwidth and delay-efficient distributed data searching in Fog-supported P2P content delivery networks," The Journal of Supercomputing, pp. 1-22, 2017. Article (CrossRef Link)

[22] Shojafar, Mohammad, Nicola Cordeschi, Enzo Baccarelli, "Energy-efficient adaptive resource management for real-time vehicular cloud services," IEEE Transactions on Cloud computing, 2016. 


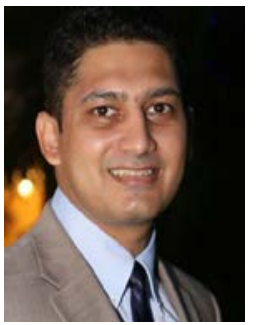

Adnan Khalid is a lecturer at the prestigious Government College University Lahore. He has an M.Phil. in Computer Science and is pursuing a Ph.D. in Cloud Computing. His area of research is Fog Computing and he intends to highlight the benefits of this relatively novel field of research.

Adnan Khalid teaches research methods and software engineering at the undergraduate and postgraduate level. He has four scholarly publications in local HEC recognized journals.

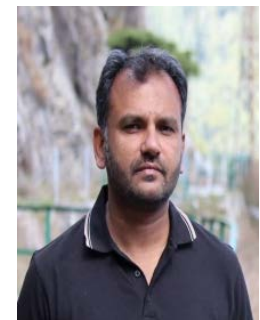

Dr. Muhammad Shahbaz is the Head of department of one of the most prestigious engineering and technology universities in Asia, University of Engineering and Technology, Lahore. He has a Ph.D in Computer Science specializing in Data Mining and Artificial Intelligence. He is an approved Ph.D supervisor and a full Professor of Computer Science. 